\title{
Effects of a Global Symmetry on the Observation of Astronomical Objects
}

\author{
Carmine Cataldo ${ }^{1}$ \\ ${ }^{1}$ Independent Researcher, $\mathrm{PhD}$ in Mechanical Engineering, Battipaglia (SA), Italy \\ Correspondence: Carmine Cataldo, Independent Researcher, PhD in Mechanical Engineering, Battipaglia (SA), \\ Italy. E-mail: catcataldo@hotmail.it
}

Received: September 9, 2016

Accepted: September 28, 2016

Online Published: September 30, 2016

doi:10.5539/apr.v8n5p75

URL: http://dx.doi.org/10.5539/apr.v8n5p75

\begin{abstract}
This paper has been drafted in an attempt to carry out an explicative discussion, as far as possible jargon-free, about the consequences related to the alleged existence of a global symmetry. The Universe in its entirety, hypothesized as being at rest, is assimilated to a four-dimensional ball. Obviously, the reality we are allowed to perceive cannot exceed the three ordinary dimensions. Each point at rest, or better what we perceive as being a point at rest, may be nothing but one of the intersections between a straight line, that crosses the center of the 4-ball, and the boundary of the ball itself. Actually, taking into account the symmetry, we could even state that each point and its antipode are to be considered as being the same thing. In reading the discussion, finalized to establish a qualitative relation between the hypothesized symmetry and the signals' transmission, it appears quite evident how a shorter line of reasoning could have been followed, so as to make the description more general and elegant: this way, however, the paper would have surely lost most of its explicative capability.
\end{abstract}

Keywords: symmetry, astronomical objects, light signals transmission, fourth dimension.

\section{Introduction}

The Universe is seen as belonging to the so called oscillatory class (Harrison, 1967). Consequently, although the beginning of a new cycle could be conventionally fixed, both a beginning and an end cannot be actually defined. We suppose, in particular, that the Universe may oscillate following a simple harmonic motion. The Hubble parameter (Hubble, 1929) may have assumed in the past, and could possibly still assume in the future, negative values. However, the variation of cosmological distances is seen as being exclusively metric: in other terms, the amount of space between whatever couple of points remains the same with the passing of time (Cataldo, 2016a). The existence of a further spatial dimension is hypothesized. The Universe in its entirety is considered as being flat, since it is assimilated to a four-dimensional ball. The corresponding boundary, on the contrary, is a curved space: this three-dimensional surface, commonly defined hypersphere, represents the universe we are allowed to perceive. Actually, the previous assertion is properly valid for any observer at rest. In fact, once fixed the center of the four-dimensional ball, whatever hypersphere, as long as it is characterized by a radius of curvature less than or equal to the one of the external surface, could represent the universe we perceive: this exclusively depends on the state of motion. On this subject, considering the symmetry in relation to the center, we have to firstly hypothesize that each point at rest and its antipode, both placed on the external surface, are actually the same thing, since belonging to the same straight line segment (Cataldo, 2016b). Now, we may suppose that as a consequence of a possible motion, any segment undergoes a radial contraction whose entity depends on the value of the tangential speed of its endpoints. The motion can be imagined as nothing but a rotation around the center of the four-dimensional ball that represents our Universe. When this happens, the endpoints are dragged, if we can say so, towards an inner hyper surface, characterized by a radius of curvature smaller than the maximum one. The value of the tangential speed acquired by the endpoints cannot exceed, in any case, that of light. Under the previous hypotheses, it is possible to assign a different meaning to the well known Lorentz transformations (Lorentz, 1904). Moreover, once reinterpreted the relativistic equations, we may even state, in a certain sense and measure, that it is possible to virtually travel faster than light. In any case, as far as this paper is concerned, we consider each point as being at rest. Although slightly off topic, it is worth underlining how, in the light of the hypotheses up until now put forward, once identified the Universe with an ideal fluid characterized by an appropriate value of the parameter that appears in the equation of state suggested by Zeldovich (Zeldovich, 
1961), the Friedmann-Lemaître equations (Friedmann, 1922) can be alternatively deduced (Cataldo, 2016c) by carrying out a specific position involving the gravitational constant (Cataldo, 2016d). This way, we no longer need to exploit the Einstein field equations (Einstein, 1916).

\section{Discussion}

Firstly, it is worth underlining, once again, how the variation of cosmological distances may not be herein considered as a real phenomenon: as a consequence, the amount of space between two generic point at rest does not undergo any modification.

Secondly, by virtue of the alleged existence of a further spatial dimension, it is necessary to specify how light signals are not to be considered as arisen from a single point. According to what hypothesized in the introduction, a generic source, as punctual as it may seem, is not actually a point, but rather a straight line segment.

The discussion will be qualitatively carried out by referring to a bi-dimensional scenario: in other terms, the universe we are allowed to perceive is portrayed as a circumference.

Let's denote with $P^{+}$and $P^{-}$the endpoints of the segment that represent the source, and with $O^{+}$and $O^{-}$the endpoints of the segment that represents the observer (Figure 1).

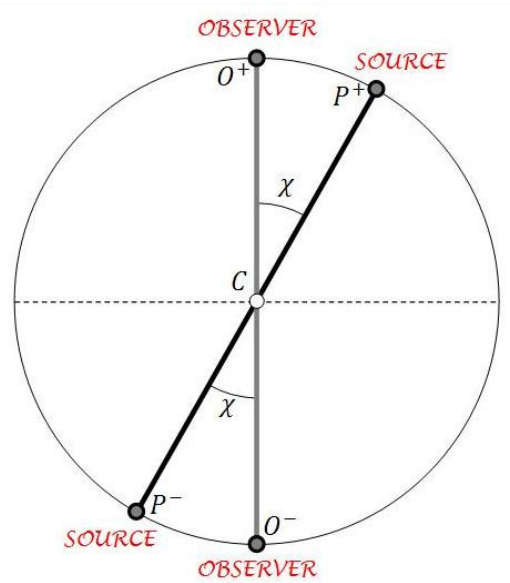

Figure 1. Observer and Light Source

When the transmission of a signal takes place, each point of the segment bordered by $P^{+}$and $P^{-}$starts sending impulses. Light travels tangentially, following the so called geodesics. In our case, since both the observer and the source are considered as being at rest, the only geodesic we have to reflect on is the circumference portrayed in the previous figure. No obstacle is placed along the path followed by the light signals.

Now, let's denote with $R$ the radius of curvature of the Universe, and with $\chi$ the angular distance between the source and the observer, both imagined as being at rest.

The corresponding arc length, denoted with $s$, can be obviously written as follows:

$$
s=R \chi
$$

To cover the above mentioned distance, light needs a time, denoted by $T$, provided by the underlying relation:

$$
T=\frac{s}{c}=\frac{R \chi}{c}
$$

As usual, the speed of light, approximately $3.00 \times 10^{8}$ meters per second in vacuum, has been denoted with $c$. In order to simplify the discussion as much as possible, let's suppose that the status of the source may be considered as a discrete variable. Moreover, let's suppose that the status may change with a frequency, denoted by $\boldsymbol{v}$, whose value is banally provided by the following relation:

$$
v=\frac{1}{T}=\frac{c}{R \chi}
$$


To make the discussion even simpler, let's consider, without a real loss of generality, the specific case of $\chi=30^{\circ}$. Moreover, in an attempt to further simplify the description, the values of the angular distances will be set as being multiples of $\chi$.

When $t=0$ (Figure 2), the status of the source is equal to one. Information starts travelling, both clockwise and counterclockwise. The observer has not yet received any information whatsoever (zero).

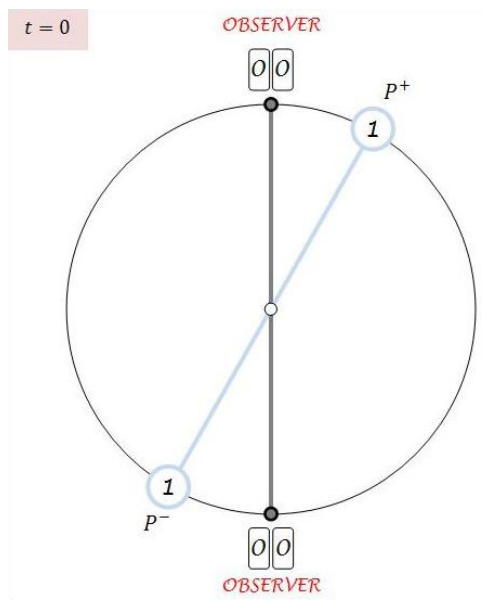

Figure 2. Scenario when $t=0$

When $t=T$ (Figure 3), the status of the source becomes instantaneously equal to two. Simultaneously, the observer receives, on the positive side, the piece of information concerning the previous status of the source (one). On the negative side, nothing has been received yet (zero). A further possible observer placed in $A$ would see the status one.

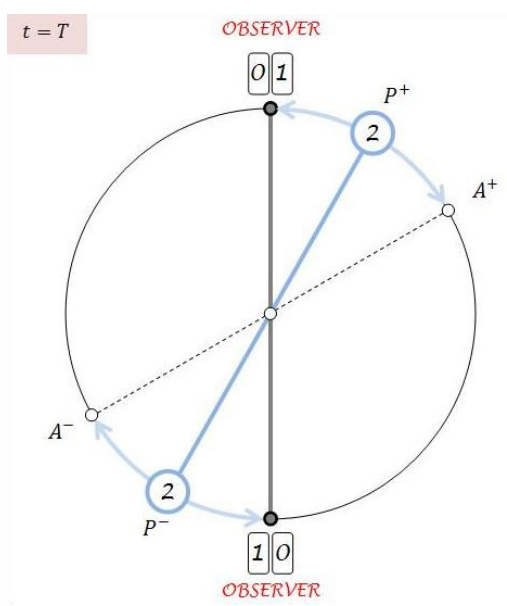

Figure 3. Scenario when $t=T$

When $t=2 T$ (Figure 4), the status switches to three. Simultaneously, the observer receives, on the positive side, the piece of information concerning the previous status $(t w o)$. On the negative side, nothing has been received yet (zero). Two possible observers placed in $A$ and $B$ would see, respectively, the statuses two and one. 


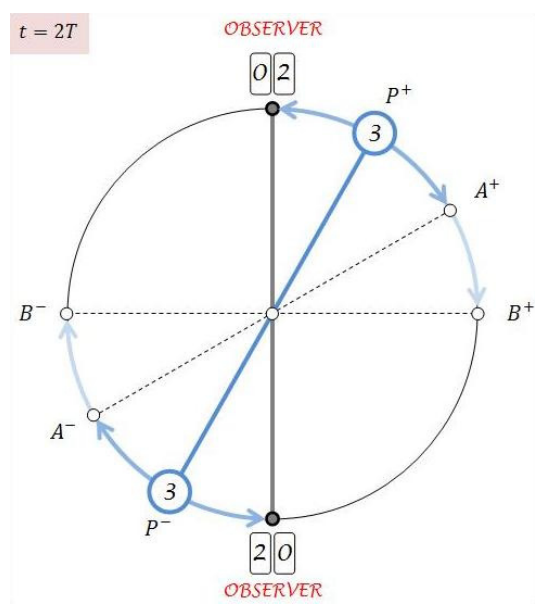

Figure 4. Scenario when $t=2 T$

When $t=3 T$ (Figure 5), the status switches to four. Simultaneously, the observer receives, on the positive side, the piece of information concerning the previous status (three). On the negative side, nothing has been received yet (zero). As for further possible observers placed along the path, from now onwards, the line of reasoning exploited in describing the previous scenario can be obviously followed.

When $t=4 T$ (Figure 6), the status switches to five. Simultaneously, the observer receives, on the positive side, the piece of information concerning the previous status (four). On the negative side, nothing has been received yet.

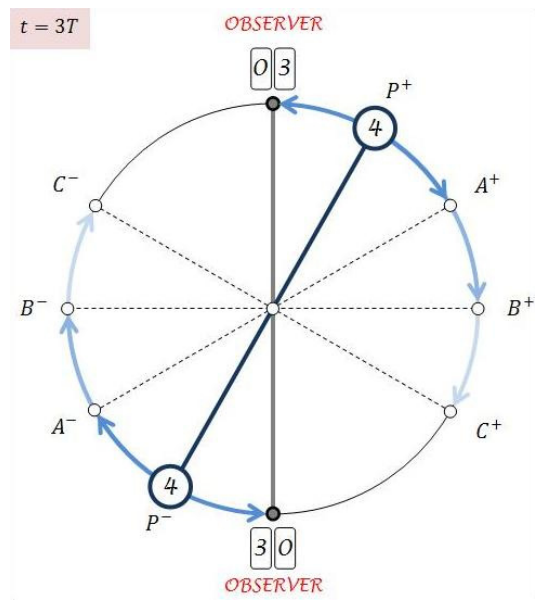

Figure 5. Scenario when $t=3 T$

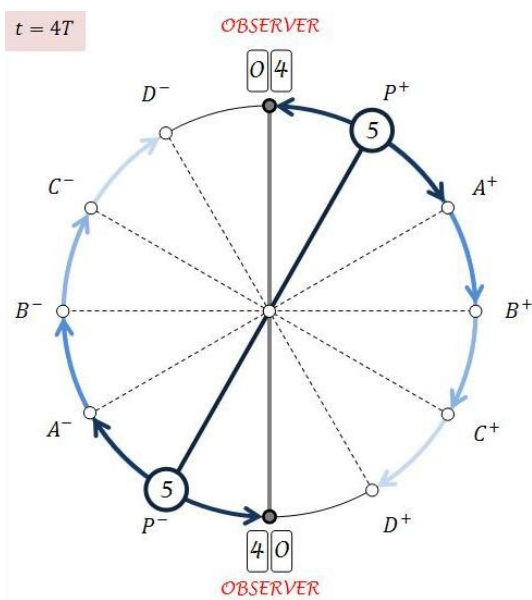

Figure 6. Scenario when $t=4 T$ 
When $t=5 T$ (Figure 7), the status switches to six. Simultaneously, the observer receives, on the positive side, the piece of information concerning the previous status of the source (five). On the negative side, this time, the observer receives the piece of information concerning the first status (one).

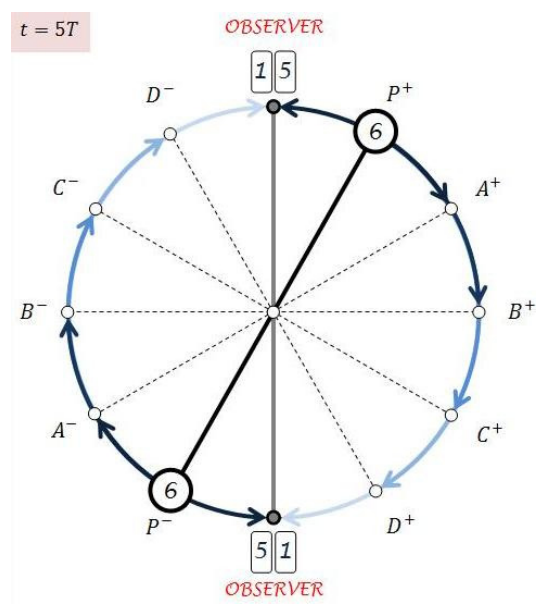

Figure 7. Scenario when $t=5 T$

\section{Conclusions}

A general overview of the fundamental characteristics of the typology of Universe we have hypothesized has been presented in the introduction: on this subject, we have tried to provide as many particulars as possible, some of which not strictly necessary to the full understanding of this paper. In an attempt to offer a description suitable even for a non expert reader, the discussion has been carried out by considering the specific case of a source that regularly switches its status. Actually, the real process is not at all discrete, and the transitions take place continuously with the passing of time. Just to qualitatively synthesize the result we have obtained, we may state that an observer receives, along the same direction, two distinct images, deeply different from each other, coming from opposite sides. Net of the evaluation of the distances, that could be obviously carried out by considering the cosmological redshift, the observer is sure to have seen two different objects. Actually, by virtue of what discussed in this paper, the images received by the observer represent two different statuses of the same object. Along the same direction, the observer sees on the one side, for example, a protostar, and on the opposite side, simultaneously, a supernova: in other terms, birth and death of the same astronomical object.

\section{References}

Cataldo C. (2016a). Further Remarks on the Oscillating Universe: an Explicative Approach. Research \& Reviews: Journal of Pure and Applied Physics, 4(3), 3-37. Retrieved from http://www.rroij.com/open-access/ further-remarks-on-the-oscillating-universe-an-explicative-approach-.pdf

Cataldo C. (2016b). Towards a New Relativity: how to Travel Faster than Light. Research \& Reviews: Journal of Pure and Applied Physics, 4(1), 7-14. Retrieved from http://www.rroij.com/open-access/towards-a-new -relativity-how-to-travel-faster-than-light-.pdf

Cataldo C. (2016c). A simplified model of Oscillating Universe: alternative deduction of Friedmann-Lemaître equations with a negative cosmological constant. Research \& Reviews: J. of Pure and App. Phys., 4(2), 1-3. Retrieved from http://www.rroij.com/open-access/a-simplified-model-of-oscillating-universe-alternative -deduction-of-friedmannlematre-equations-with-a-negative-cosmological-consta-pdf

Cataldo C. (2016d). Beyond the Metrics: qualitative introduction to the Modified Gravitation. Research \& Reviews: Journal of Pure and Applied Physics, 4(2). http://www.rroij.com/open-access/beyond-the-metrics -qualitative-introduction-to-the-modified-gravitation-.pdf

Einstein A. (1916). Relativity: The Special and General Theory (translated by Lawson RW, 1920). Henry Holt and Company, New York. Retrieved from https://archive.org/details/cu31924011804774

Friedmann A. (1922). Über die Krümmung des Raumes. Zeitschrift für Physik, 10, 377-386. English Translation (1999) On the Curvature of Space. General Relativity and Gravitation, 31(12), 1991-2000. Retrieved from http://www.ymambrini.com/My_World/History_files/Friedman_1922.pdf 
Harrison E. R. (1967). Classification of uniform cosmological models. Monthly Notices of the Royal Astronom. Society, 137, 69-79. Retrieved from http://paperity.org/p/39522229/classification-of-uniform-cosmological -models

Hubble E. (1929). A Relation between Distance and Radial Velocity among Extra-Galactic Nebulae. Proceedings of the National Academy of Sciences of the United States of America, 15, 168-173. Retrieved from http://www.ncbi.nlm.nih.gov/pmc/articles/PMC522427/pdf/pnas01016-0006.pdf

Lorentz H. A. (1904) Electromagnetic phenomena in a system moving with any velocity smaller than that of light. Proceedings of the Royal Netherlands Academy of Arts and Sciences, 6, 809-831. Retrieved from http://www.orgonelab.org/EtherDrift/Lorentz1904.pdf

Zeldovich Y. (1961). The equation of state at ultrahigh densities and its relativistic limitations. J. Exptl. Theoret. Phys. (U.S.S.R.), 41, 1609-1615. Retrieved from http://www.jetp.ac.ru/cgi-bin/dn/e_014_05_1143.pdf

\section{Copyrights}

Copyright for this article is retained by the author(s), with first publication rights granted to the journal.

This is an open-access article distributed under the terms and conditions of the Creative Commons Attribution license (http://creativecommons.org/licenses/by/4.0/). 